\title{
Quasi-binarity of massive stars in young dense clusters - the case of the Orion nebula cluster
}

\author{
S. Pfalzner and C. Olczak \\ I. Physikalisches Institut, University of Cologne, Zülpicher Str. 77, 50937 Cologne, Germany \\ e-mail: pfalzner@ph1.Uni-koeln.de
}

Received 10 August 2007 / Accepted 25 September 2007

\begin{abstract}
Context. Observations indicate that in young stellar clusters the binary fraction for massive stars is higher than for solar mass stars. For the Orion Nebula Cluster (ONC) there is a binary frequency of $\sim 50 \%$ for solar-mass stars compared to $70-100 \%$ for the massive $\mathrm{O}$ - and B-stars.

Aims. We explore the reasons for this discrepancy and come up with two possible answers: a) a primordially higher binarity of massive stars could be inherent in the star formation process or b) the primordial binary rate might be the same for solar-mass and massive stars, but the higher capture cross-section of the massive stars possibly leads to the formation of additional massive binaries in the early cluster development. Here we investigate the likelihood of the latter scenario in detail using the ONC as an example.

Methods. $N$-body simulations are performed to track the capture events in an ONC-like cluster.

Results. We find that, whereas low-mass stars rarely form bound systems through capture, the dynamics of the massive stars especially in the first $0.5 \mathrm{Myr}$ - is dominated by a rapid succession of "transient binary or multiple systems". In observations, the transient nature of these systems would not be apparent, so that they would be classified as binaries. At 1-2 Myr, the supposed age of the ONC, the "transient" massive systems become increasingly stable, lasting on average several $10^{6}$ yr. Despite the ONC being so young, the observed binary frequency for massive stars - unlike that of solar-mass stars - is not identical to the primordial binary frequency, but is increased by at least $10-15 \%$ through dynamical interaction processes. This value might be increased to at least $20-25 \%$ by taking disc effects into account.

Conclusions. The primordial binary frequency could well be the same for massive and solar mass stars because the observed difference can be explained by capture processes alone.
\end{abstract}

Key words. binaries: general - galaxies: clusters: general - galaxies: clusters: individual: ONC - methods: numerical

\section{Introduction}

Observations made predominantly in the 1990's found the fraction of field stars in multiple systems to be high: $\sim 55 \%$ for solar-mass stars (Duchquennoy \& Mayor 1991) and 35-42\% for M-dwarf low-mass stars (Fischer \& Marcy 1992; Reid \& Gizis 1997). To explain the high binary rate of field stars, gravitational capture between two single stars was initially discussed as a possibility. However, it was acknowledged that while some binaries might actually form this way, capture cannot be the primary binary formation process, due to the low likelihood of such an event.

Observations of pre-main sequence stars support the theory that binaries are already formed during star formation. The multiplicity of young pre-main-sequence (PMS) stars in many regions (especially in loose T-associations like Taurus) seems to be systematically higher than that of their main-sequence counterparts (e.g. Mathieu et al. 2000, and references therein). Currently, fragmentation of the molecular cloud during the formation of protostars is the accepted explanation for the formation of a binary or multiple star system (Boss 1992; Tohline et al. 1999)

However, unsolved problems remain. Denser young stellar clusters like the central (very dense) Trapezium cluster of the Orion Nebula Cluster, IC 348 and NGC 2024, show much lower binary frequencies than the above-mentioned loose T-associations (Prosser et al. 1994; Padgett et al. 1997;
Bouvier 1997; Patience et al. 1998; Petr 1998; Simon et al. 1999; Scally et al. 1999; Duchêne et al. 1999; McCaughren 2001; Beck et al. 2003; Liu et al. 2003; Luhman et al. 2005; Kraus \& Hillenbrand 2007). There is emerging evidence that high-density regions might have lower binary frequencies or preferentially smaller binary separations (Köhler et al. 2006). Essentially, two theoretical concepts exist to explain this observational finding: (a) the dynamical disruption of wide binaries (with a separation distance $\left.r_{\mathrm{s}}>100 \mathrm{AU}\right)$ through close stellar encounters decreases the primordial binary fraction in dense clusters (Kroupa 1995; Kroupa et al. 1999) or (b) the influence of the temperature of the molecular cores on the fragmentation mechanism results in higher primordial binary fractions (Durisen \& Sterzik 1994; Sterzik et al. 2003) in less dense clusters.

Possibly connected with this problem is the question that is addressed here: why does the binary frequency increase from $\sim 50 \%$ for solar-type field stars (Duchquennoy \& Mayor 1991; Fischer \& Marcy 1992) to 70\% for massive O- and B-stars (Abt et al. 1990; Mason 1998)? In this paper we consider the possible reasons for the higher binary frequency of massive stars in comparison to intermediate mass stars. In principle there are only two possibilities: either massive stars are more likely to be binaries to start with, or their binarity increases within the first Myr of their existence in a significant way. In other words, the first possibility would mean that the star formation process is mass-dependent. The question of whether this is true is very difficult to answer, since there are still problems in explaining how 
even a single star more massive than $\sim 10 M_{\odot}$ can form (for a review see Yorke \& Zinnecker 2007). Here we restrict ourselves to the second possibility, i.e. we start with the assumption that the primordial number of binaries is the same for solar-mass and massive stars, and ask whether dynamical processes can lead to a sufficient amount of additional binaries to explain the difference in observed binary frequency between solar-mass and massive stars.

To do this we revisit capture processes as a potential candidate for part of the binary formation. In the early 90s, stellar dynamics simulations (Clarke \& Pringle 1991) of the ONC showed that dynamical interactions in a cluster cannot form a significant enough number of binaries from an initially single-star population to explain a binary population of $50 \%$ or more. Indeed, we also find that for solar-type stars, capture processes rarely lead to bound systems. However, it will be demonstrated here that the situation is different for massive stars. The combination of the higher capture cross-section of the massive stars with the fact that they are predominantly located in the high density central regions of the cluster leads to the formation of massive binaries in addition to the primordial existing ones, by capture processes in the early cluster development.

As a model cluster we chose the Orion Nebula Cluster, because it is one of the densest close-by clusters in the Galaxy, so if capture processes play any role one should find indications for it here. In addition, it is observationally one of the best investigated clusters, so the detailed knowledge of its properties helps to limit the simulation parameters. What is known about the binary statistics in the ONC? In Orion the binary rate for solar-type stars is $\sim 50 \%$ whereas the binary rate for massive stars is $\sim 75 \%$ (Preibisch et al. 1999; Köhler et al. 2006). The latter value has a high uncertainty upwards, as observations of the binary frequency of high-mass stars are usually obtained from small-sized samples, and heterogeneity of the sample and/or selection effects in general cannot be completely excluded. Correction for completeness, as done for lower mass stars, is difficult as the necessary assumptions of the binary mass ratio and orbital period distribution cannot be made. This means that the observations could be equally well be interpreted as a $100 \%$ binary rate for massive stars in the ONC (Preibisch et al. 1999; Köhler et al. 2006). It is possible that there exists a trend of decreasing multiplicity from the centre of the ONC outwards (Preibisch et al. 1999; Köhler et al. 2006). One concludes that differences in the formation mechanisms of high-mass and low-mass stars multiple systems must exist.

Although capture is usually no longer considered, Moeckel \& Bally (2007a), studying single isolated encounter events of disc-surrounded stars only, suggested that encounter-induced capture could be a common event for massive stars. They estimated that the density in the ONC would suffice for capture processes alone to lead to $50 \%$ binaries. Although we do not include the effect of discs in the simulations presented here, we perform the first systematic study of the stellar dynamics in an ONClike system with the emphasis on the massive stars. Our study reveals that massive stars frequently form "transient bound systems" (TBS) through gravitational interaction. Single transient bound systems are indistinguishable from long-lasting classical binaries in observations, but in Sect. 4 we will see that some binary statistics results could be interpreted as the effect of a population of capture-formed massive binaries.

What kind of binaries form? Binaries can be sub-divided into three dynamical groups according to the ratio between $E_{\mathrm{b}}$, the binding energy of the binary, and $E_{\text {kin }}$, the kinetic energy of the encounter with another star of the system: (i) the wide, or soft,

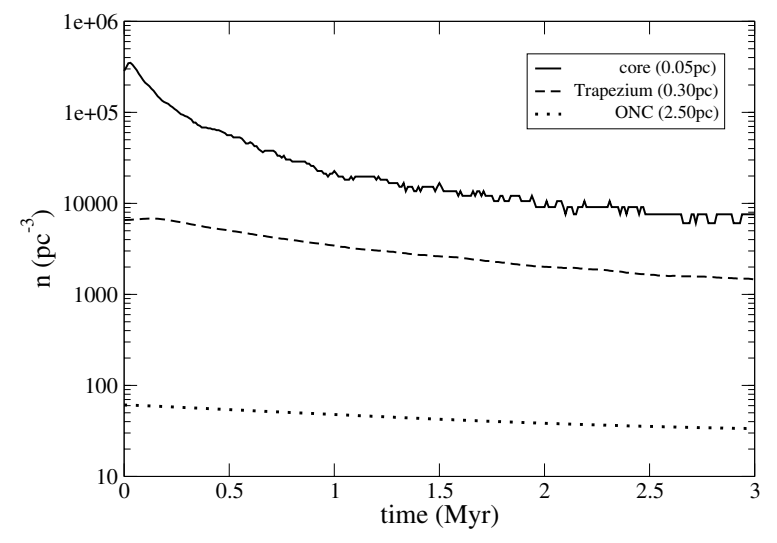

Fig. 1. The development of the particle density as a function of time for the central core, the Trapezium region and the entire ONC.

binaries with $E_{\mathrm{b}} / E_{\mathrm{kin}}<1$, (ii) the dynamically active binaries $E_{\mathrm{b}} / E_{\text {kin }} \sim 1$, and (iii) the tight or hard binaries $E_{\mathrm{b}} / E_{\text {kin }}>1$. Soft and hard binaries are relatively well understood (see Heggie \& Hut 2003, for a review), and Heggie (1975) and Hills (1975) previously summarised the dynamics of these systems with the observation that "soft binaries soften and hard binaries harden". Amongst these groups, the active binaries with intermediate binding energies are the least well understood. Such binaries couple efficiently to the cluster, and exchange energy with it. We will see that it is this type of binary that the massive stars in the ONC primarily form in the initial phases of the cluster development. These active binaries or TBS are quite proficient in exchanging partners.

Section 2 describes the numerical method applied. In Sect. 3 we determine the number of TBS formed by capture in the ONC, followed by a detailed analysis of their properties. In Sect. 4 we discuss how these findings might change the interpretation of the origin of a higher binary rate for massive stars.

\section{Method}

For simplicity we start with a system initially consisting only of single stars; the influence of both primordial binaries and discs around the stars are excluded from this first study. Primordial binaries would make it more difficult to track the effect of the capture processes themselves, we will discuss in Sect. 4 how the assumption of there being no primordial binaries effects our results. The influence of discs can only be included if detailed knowledge of star-disc-star-disc interactions leading to binaries exists for the entire parameter space required. To our knowledge, only single cases of such interactions have been modelled so far.

In the cluster simulations we followed the dynamical development of $\sim 4000$ stars in a virial equilibrium situation, i.e. $Q_{\text {vir }}=0.5$, with a spherical density distribution $\rho(r) \sim r^{-2}$ using NBODY6 ${ }^{++}$(Aarseth 2003; Spurzem 1999). Gas components and the potential of the background molecular cloud OMC 1 were neglected in these simulations. The most massive star was assigned a mass $M^{*}=50 M_{\odot}$ and all other stars' masses were assigned according to the mass distribution given by Kroupa (2001),

$\xi\left(M^{*}\right)= \begin{cases}0.035 M^{*-1.3} & \text { if } 0.08 \leq M^{*}<0.5 \\ 0.019 M^{*-2.2} & \text { if } 0.5 \leq M^{*}<1.0 \\ 0.019 M^{*-2.7} & \text { if } 1.0 \leq M^{*}<\infty\end{cases}$

The simulations were performed as described in Olczak et al. (2006). There, the quality of the dynamical models was judged 
a)

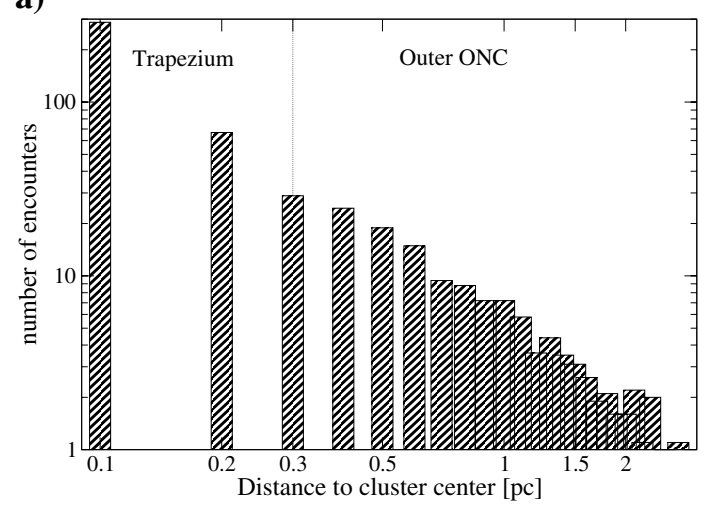

b)

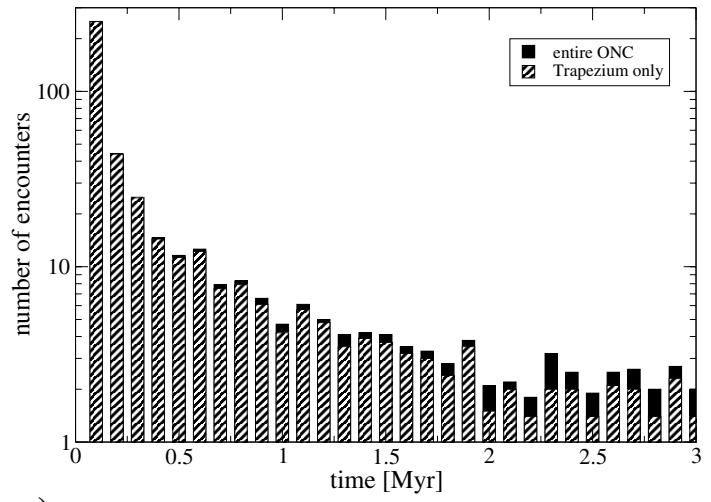

c)

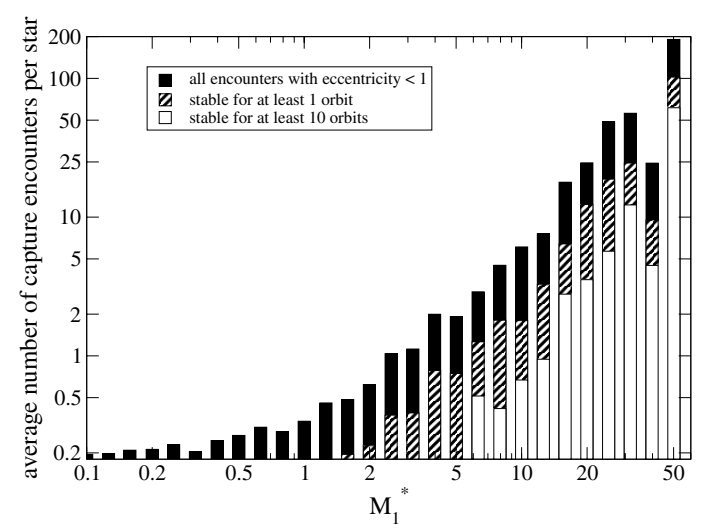

Fig. 2. Number of encounters with $\epsilon<1$ as function of a) radial distance to cluster centre, b) time and c) primary mass $\mathrm{M}_{1}^{*}$. In b) the number of encounters in the Trapezium and the entire ONC are shown. In c) all encounters with $\epsilon<1$ independent of the duration of the bound state are shown, but although the ones that keep at least bound for one (dashed line) and ten (dotted line) orbits.

by comparing them to observational data at $1-2 \mathrm{Myr}$, marking the range of the mean ONC age. The quantities of interest were: number of stars, half-mass radius, number densities, velocity dispersion and projected density profile. In the initial cluster the central number density within $0.05 \mathrm{pc}$ was $2.85 \times 10^{5} \mathrm{pc}^{-3}$ and the velocity dispersion $2.4 \mathrm{~km} \mathrm{~s}^{-1}$. There is a fast development of the number density (see Fig. 1) which peaks in the core at $\sim 0.03$ Myr.

However, we found it necessary to improve the tracking of the cluster centre for comparison to observational data. Previously we used the centres-of-mass to define the cluster centre. However, as the simulations contain only a limited number of stars, "escapers" kicked out of the cluster due to strong interactions can temporarily shift the centre-of-mass before they are removed at large distances. Using the mass density or number density centre is a more reliable alternative, especially since observers usually use either the brightness concentration or the maximum projected number density of objects as a reference point. However, since the local particle density changes strongly with time, so does the estimated density centre, which is additionally influenced by the choice of the number of nearest neighbours to some degree.

Therefore we use the density centre, but introduce the following smoothing algorithm: instead of using all simulation particles to define the centre of mass, particles at large distances from the cluster centre are excluded. On the other hand, this subsample has to be large enough that proper statistics are possible, and that the most massive stars are not too dominant. This is achieved in practice by requiring that the sub-sample contain at least $10 \%$ of the total stellar population, and that the most massive star must represent at most $10 \%$ of the mass of this subsample. The centre-of-mass of the sub-sample serves as the new cluster centre. This algorithm combines the advantages of keeping the cluster centre close to the density centre and taking a larger stellar sample into account to smooth the strong temporal fluctuations.

Having defined the cluster centre in such a way, simulations were selected as valid representations of the ONC if they fulfilled the following criteria:

- the projected density distribution at $1 \mathrm{Myr}$ has to match the data from Hillenbrand \& Hartmann (1998) and McCaughrean et al. (2002) within the statistical errors;

- the most massive star, $\theta^{1} \mathrm{C}$ Ori, must be located inside the Trapezium Cluster $\left(R_{\mathrm{TC}}=0.3 \mathrm{pc}\right)$ for at least $1 \mathrm{Myr}$, the estimate of the mean age of the ONC.

Altogether we have selected 40 simulations according to the above criteria. During the course of these simulations we track all encounters with eccentricity $\epsilon<1$, noting the stellar mass ratio, periastron, eccentricity and duration of the bound state. These parameters were not checked for bound systems with more than two partners, as we want to concentrate on the binary rather than the general multiplicity rate in this investigation.

Despite the large number of simulations and the resulting several hundred TBS formed per simulation run, one still has to deal with small-number statistics here. There are two reasons for this: the parameter space in mass ratio, periastron, etc. is very large and it is often necessary to restrict the sample to a certain time interval, or to certain systems, to stay stable for a certain duration. This leads to some difficulty, in that the results in the following section are sometimes presented for different time-intervals. We only present results where we have at least 20 events per bin to guarantee statistical significance. One should keep in mind that observations usually have much fewer objects to work with when determining binary properties.

\section{Results}

All encounter events with an eccentricity $\epsilon<1-$ hitherto called "capturing encounters" - are recorded. We find that these capturing encounters mostly happen early on in the cluster development, close to the cluster centre, and mainly involve one of the most massive stars (see Figs. 2a-c). This is exactly what one expects; close to the cluster centre the stellar density is highest, even more so early on when the cluster initially contracts (see Fig. 1) and the most massive stars dominate this area gravitationally. Specifically, during the first Myr there are on average 


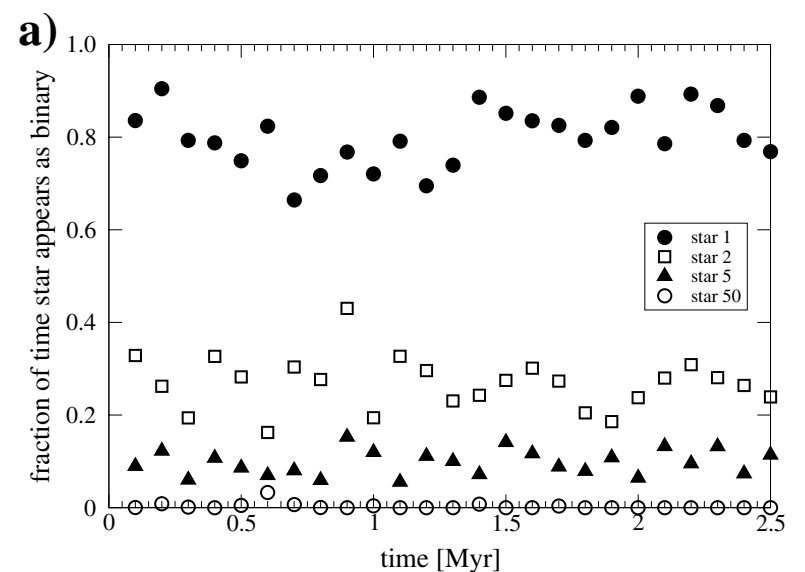

b)

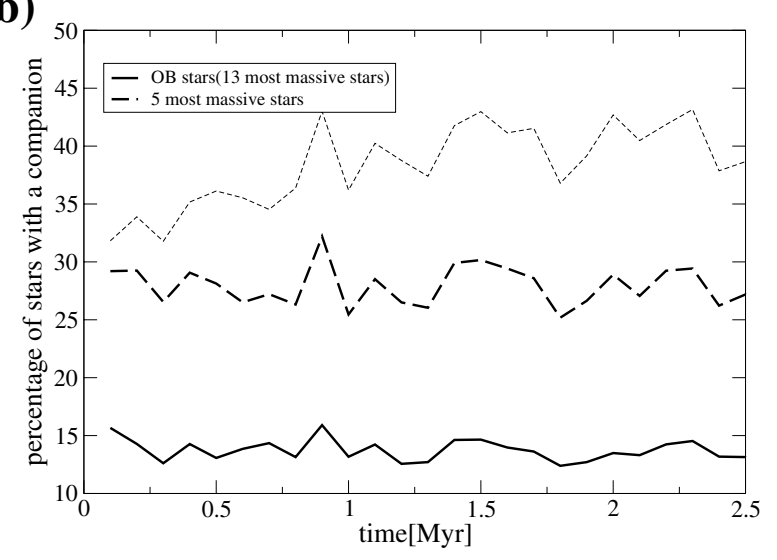

Fig. 3. a) shows the fraction of time a TBS would appear as a binary for different primary stars. The distribution of the stellar masses is not fixed, but varies. This is done to give a better representation of the IMF on average. Therefore no fixed stellar masses can be given here, but the higher star number is equivalent to lower stellar mass. In b) the percentages of the 5 (dashed line) and 13 (full line) most massive stars are shown, which are part of an encounter-induced binary in a time interval of $0.1 \mathrm{Myr}$, as a function of cluster age. The thin dashed line shows the case where these massive TBS are considered twice.

more than 350 capturing encounters in the Trapezium region, in contrast to $\sim 150$ in the rest of the ONC, which covers a thousand times larger spatial volume (see Fig. 2a). During the first $0.3 \mathrm{Myr}$ of the cluster development more than 300 capturing encounters occur; afterwards, less than 15 capturing events happen in any 0.1 Myr time interval (see Fig. 2b). Whereas the most massive star has on average approximately 200 capturing encounters in the first $5 \mathrm{Myr}$, a star with $M_{1}^{*}=1 M_{\odot}$ has less than one. In addition, a higher percentage of the capturing encounters involving the massive stars leads to relatively stable configurations. If the primary is of mass $M_{1}^{*}=50 M_{\odot}$, about half of the forming systems stay stable for at least one orbit, and a quarter for at least 10 orbits. In contrast, for a primary of mass $M_{1}^{*}=1 M_{\odot}$ about a third of the forming TBS stay stable for one orbit and less than $10 \%$ for more than 10 orbits. Here, and in the following, the word "primary" is used for the more massive rather than the more luminous star, in contrast to convention in observations.

We are now in a position to ask which binarity would be observed for clusters like our ONC model cluster. Figure 3a shows the probability of finding the most massive stars in a bound state as a result of a capturing process. It can be seen that the most massive star would, in the majority of cases, be found as a partner in a bound state. The second most massive star would be likely to be bound in $\sim 30 \%$ of all cases. For stars with lower mass the likelihood to be found in such a capture-induced bound state decreases rapidly with decreasing mass (see star 5 and 50 in Fig. 3a, where higher numbers are equivalent to lower masses).

Figure $3 b$ shows the likelihood of the 5 and 13 (number of $\mathrm{OB}$ stars in the ONC) most massive stars of the cluster to have at least one companion (here the percentage is shown) as a function of time. At 2 Myr 10-15\% of all the OB stars and $25-30 \%$ of the five most massive stars would form TBS due to the interaction dynamics in the cluster, and would appearing as binaries. Here, a TBS is taken into account only once if both stars were massive. The thin dashed line in Fig. $3 b$ shows the case where these massive TBS are considered twice. This is equivalent to the likelihood of a specific star being in a TBS. For the five most massive stars this likelihood of being in a TBS is $\sim 40 \%$ at 2 Myr. Figure $3 b$ agrees with the result by Moeckel \& Bally (2007b) who found an expected binary fraction of $12 \%$ for a star with $M_{*}=20 M_{\odot}$ at $0.5 \mathrm{Myr}$. So, for the most massive stars of the cluster there is a high likelihood of it being a TBS and appearing as a binary. The number of TBS is actually sufficient to explain the difference in binary rates between massive and solar-mass stars. But what are the properties of these capture-formed TBS?

We start with the periastra of the transient bound systems. Figure 4a shows the average periastron as a function of the primary mass $M_{1}^{*}$. The values are averaged over the first 5 Myr of the cluster development. It can be seen that the average periastron declines with increasing primary mass from $\sim 700 \mathrm{AU}$ for stars with $M_{1}^{*}<0.1 M_{\odot}$, to $\sim 60 \mathrm{AU}$ for $M_{1}^{*}=50 M_{\odot}$, reflecting the fact that massive stars form tighter bound systems more easily. The decline in periastron can be approximated by a linear dependence on $\log 10\left(M_{1}^{*}\right)$.

Figure $4 \mathrm{~b}$ shows the average periastron as a function of the mass ratio $q=M_{2}^{*} / M_{1}^{*}$ for the most massive star for the time interval 0.3-3.0 Myr. Due to the random selection process for the stellar masses in the initial cluster set-up, the second most massive star usually has a mass in the range of 30-40 $M_{\odot}$, so the statistics become increasingly worse for $q>0.7$. To a good approximation, the average periastron increases linearly with higher mass ratios from $\sim 50-65$ AU for $q=0.1$ to $\sim 160 \mathrm{AU}$ for $q=0.7$. This reflects the fact that lower mass stars can only replace a more massive star if they orbit on a smaller semimajor axis. In other words, the more equal the masses of the stars bound to each other, the larger the periastron of a relatively stable configuration can be.

The average periastron as a function of cluster age is shown in Fig. 4c. Generally there is a wide spread in periastra data obtained in single simulation runs. TBS with large periastra are usually of short duration, so it seems more appropriate to average over the periastron weighted by the duration of the bound state. This reflects the observations that are more likely to detect long-lasting bound systems than short ones. The average periastron of the $50 M_{\odot}$-star strongly depends on the cluster age, decreasing from $\sim 500-600 \mathrm{AU}$ at $0.1 \mathrm{Myr}$ to $\sim 20-30 \mathrm{AU}$ at $5 \mathrm{Myr}$. Exchange processes lead to increasingly stronger bound TBS over time. For the stars in the mass group $M_{1}^{*} \sim 30-40 M_{\odot}$ the trend is less pronounced, decreasing from $\sim 700 \mathrm{AU}$ at $0.1 \mathrm{Myr}$ to $\sim 350-450 \mathrm{AU}$ at $5 \mathrm{Myr}$. For lower mass stars the periastron does not significantly alter over the considered timespan, and is larger for lower primary masses. At 1-2 Myr, the most likely age of the ONC, the average periastron of the most massive TBS is between 50-200 AU.

Next we consider the two properties that observations usually provide: the average mass ratio $q=M_{2}^{*} / M_{1}^{*}$ and the binary frequency. 
a)

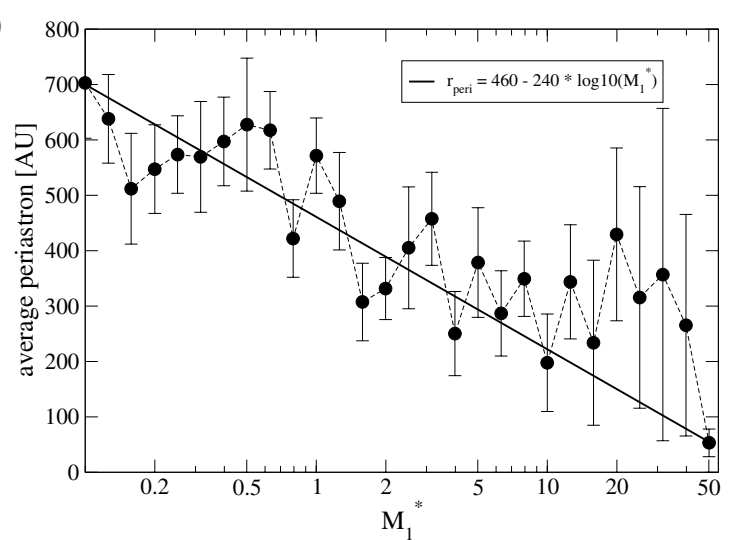

b)

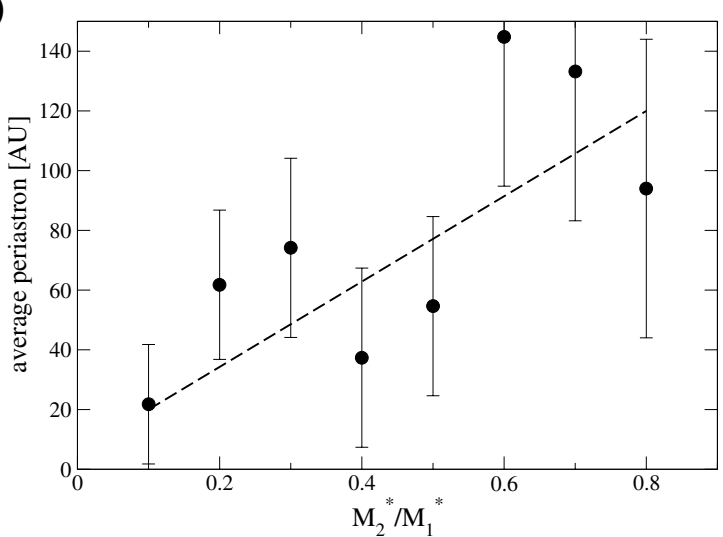

c)

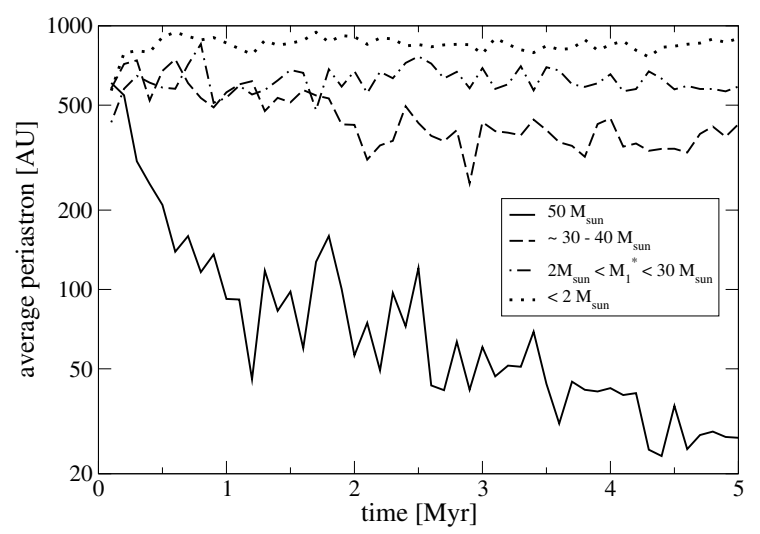

Fig. 4. Average periastron as a function of a) the primary mass $M_{1}^{*}$ averaged over all times up to $2 \mathrm{Myr}$, b) the mass ratio $q=M_{2}^{*} / M_{1}^{*}$ examplarily for the time interval $0.3-3.0 \mathrm{Myr}$ and c) time for different primaries separately: $M_{1}^{*}=50 M_{\odot}, 30 M_{\odot}<M_{1}^{*}<40 M_{\odot}, 2 M_{\odot}<M_{1}^{*}<30 M_{\odot}$, and $M_{1}^{*}<2 M_{\odot}$. Only encounters where considered were the binary remained bound for at least one entire orbit.

Figure 5a shows the average relative mass ratio $M_{2}^{*} / M_{1}^{*}$ of the TBS for the most massive stars. It can be seen that in the encounter-averaged case the relative mass of the companion increases from very low values $\left(<0.1 M_{1}^{*}\right)$ in the early cluster development to $q$-values $\sim 0.4$ at $3 \mathrm{Myr}$. If we weight the q-values by the encounter duration, the general trend remains the same but shifts to somewhat higher values. Our simulations show, in addition, the difference in this development for primaries with low and high mass. Looking at the distribution of companion masses, one sees in Fig. $5 \mathrm{~b}$ that for the most massive star at early times $(t<0.2 \mathrm{Myr})$ mass ratios $q<0.1$ dominate, whereas capturing encounters with more massive stars become increasingly
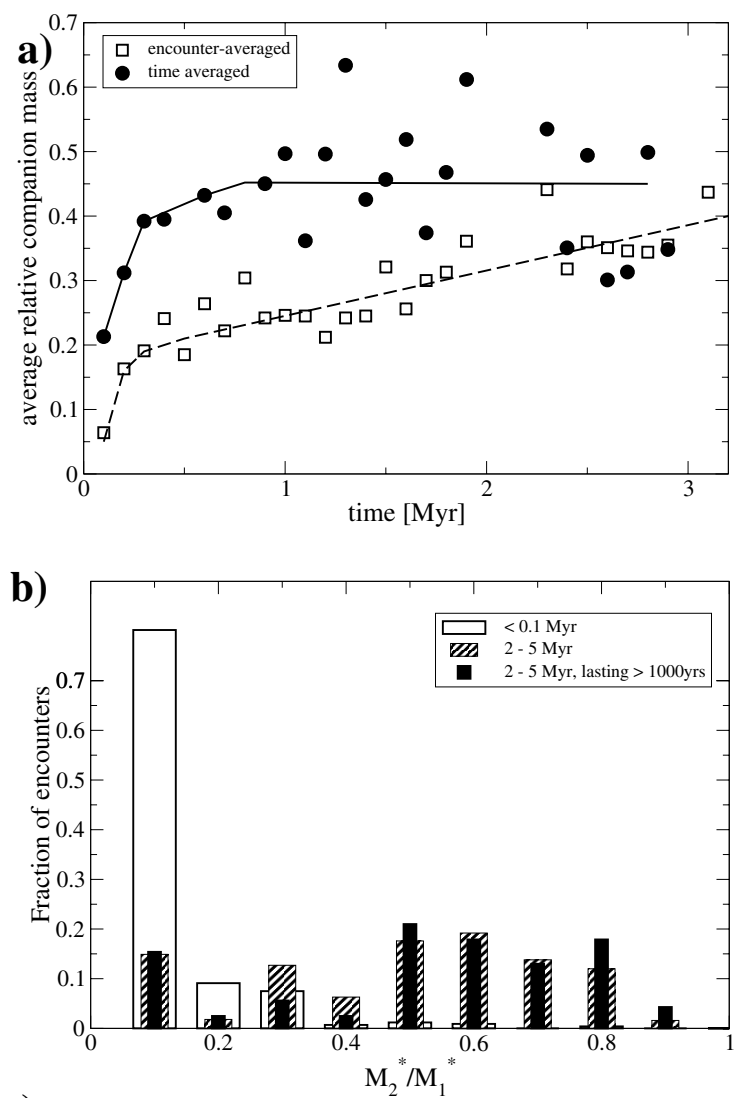

c)

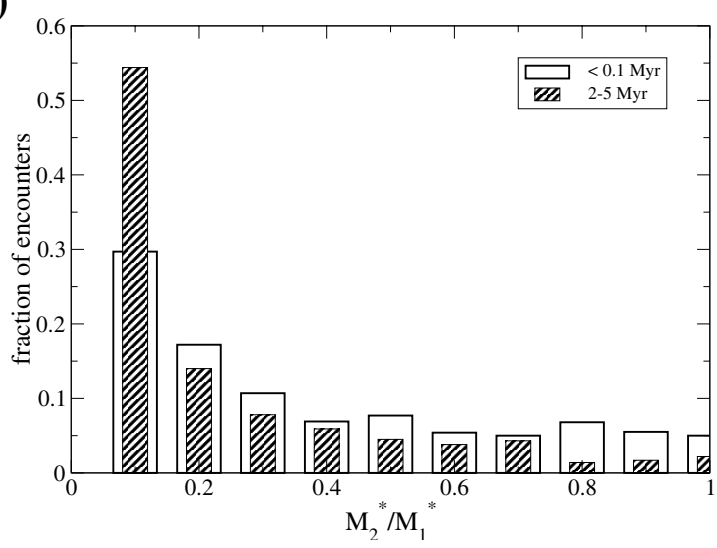

Fig. 5. a) The average relative companion mass $M_{2}^{*} / M_{1}^{*}$ as a function of time for capturing encounters for $M_{1}^{*}=50 M_{\odot}$. The line is no proper fit, but just meant as a guide line. b) Distribution of companion mass up to $0.1 \mathrm{Myr}$ and 2-5 Myr for the most massive star and c) for stars with $M_{1}^{*}<10 M_{\odot}$.

important later on in the cluster development. The mass ratio in these TBS develops from an initial preference of low-mass companions to companions with high mass. At a cluster age of 2-5 Myr the maximum of $q$ lies in the range of $0.6-0.8$, which is even more pronounced if we consider only TBS that last at least $1000 \mathrm{yr}$. The value of $q$ is not the expected equal mass binary value of 1 , because the mass of the second most massive star varies in the different simulations between $30-40 M_{\odot}$. In addition, the most massive star usually captures just one of the 10 most massive stars as a companion, and not necessarily the second most massive star.

This result of high q-values later on in the cluster development correlates very well with the observations that show that, for the massive stars, the mass ratio $q$ seems to be much smaller 


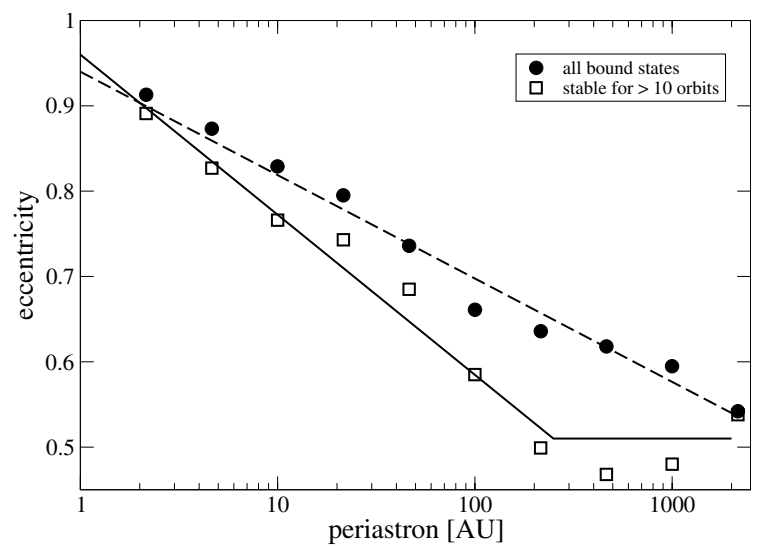

Fig. 6. Eccentricity as a function of periastron. Here only encounters where the binary remained bound for at least one or at least 10 entire orbits were considered. These are averaged over all times up to $2 \mathrm{Myr}$. Only $M_{2}^{*} / M_{1}^{*}<1$ are considered. The lines are not a proper fits, but just meant as a guide lines.

for stars in young cluster than for field stars (Goodwin et al. 2006). How quickly this development happens will depend crucially on the cluster properties such as its density.

What about the eccentricity in these bound systems? Figure 6 shows a very clear connection between eccentricity and periastron: the larger the periastron, the smaller the average eccentricity. This is simply what one would expect as a consequence of two-body relaxation. Obviously, whereas a binary with a 1 AU periastron can tolerate a 0.9 eccentricity, a binary with a $2000 \mathrm{AU}$ periastron has, on average, only a 0.55 eccentricity in the systems investigated here. If we consider only systems remaining bound for at least 10 orbits, the decline is even faster, but it levels off at about $200 \mathrm{AU}$, at an eccentricity of $\sim 0.5$. This faster decline reflects the fact that large periastron TBS only stay stable if their eccentricity is low, otherwise they are likely to undergo strong interactions with the other cluster stars. We saw before that the TBS at 1-2 Myr have, on average, a periastron of 50-200 AU. Using Fig. 6 this is coupled to an average eccentricity $\epsilon \sim 0.5-0.6$.

The trend towards more stable configurations, especially for the most massive stars, is most dramatically reflected in the average duration of TBS. The dependence of the average duration of a bound state on the stellar mass $M_{1}^{*}$ of the primary can be seen in Fig. 7. This is most pronounced for stars with $M_{1}^{*}>10-20 M_{\odot}$, where the bound state lasts on average several times $10^{6} \mathrm{yr}$, in contrast to bound state durations of on average $<10^{5} \mathrm{yr}$ for lower mass stars. Therefore, apart from the very early stages of the cluster development $(<0.1 \mathrm{Myr})$, these systems stay stable for many orbital periods.

The duration of the binary phase $t_{\text {bound }}$ increases for larger $M_{2}^{*} / M_{1}^{*}$; these are the more stable configurations that are formed later in the cluster development. For the same reason, we see in our simulations an increase of $t_{\text {bound }}$ with larger periastra and smaller eccentricities (a strong increase for $\epsilon<0.2$ ). This formation of more stable configurations is reflected by a dramatic increase of the average duration of a bound state with cluster age. Whereas bound states last on average only a few 10-100 yr at $t_{\text {cluster }}=0.1 \mathrm{Myr}$, they rise to a few $10^{6} \mathrm{yr}$ at $t_{\text {cluster }}=2 \mathrm{Myr}$. Therefore, in very young clusters these massive stars would $a p$ pear as binaries, but are actually just running through a succession of TBS.

As the duration of the bound state is a strong function of time, so is the number of orbits. It is initially higher, but

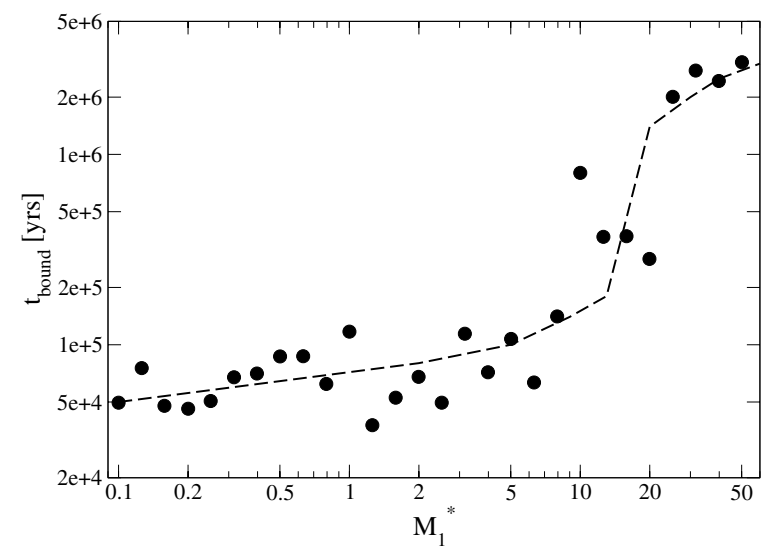

Fig. 7. Average duration of a bound state, $t_{\text {bound }}$, as a function of the primary mass $M_{1}^{*}$. The line is not a proper fit, but just meant as a guide line.

increases less rapidly for lower mass primary stars. Thus after $1 \mathrm{Myr}$, the binaries involving the most massive stars stay bound for a larger number of orbits $(\sim$ several 1000$)$.

\section{Discussion and conclusions}

The results of our simulations of capturing processes in an ONClike system can be summarized by the following: in cluster environments similar to the ONC, massive stars have a much higher probability of involvement in a capturing encounter than solarmass stars. Assuming a cluster age of 1-2 Myr, at least $10-15 \%$ of the OB stars in the ONC are in a bound state caused by capturing processes, with the average periastron of the so-formed TBS being $r_{\mathrm{p}} \sim 50-200 \mathrm{AU}$, the mass ratio $q \sim 0.4-0.5$ and the eccentricity $\epsilon \sim 0.6$. In observations, these bound systems would be regarded as normal binaries, even if the bound state is of short duration $\left(\sim 10^{3}-10^{6} \mathrm{yr}\right)$.

We have to discuss two questions: i) is the number of TBS formed sufficiently high to explain the difference in binary frequency between solar-type and massive stars and ii) are the observed properties of these TBS in accordance with observations?

For simplicity the simulations presented here started with all stars initially being single. In reality, there exists a primordial binary fraction. We saw that for massive stars, capture processes quickly lead to additional bound systems, so that there are two binary populations present - the primordial binaries and the TBS formed by capture processes. As our simulations show that at least $10-15 \%$ of the OB stars in the ONC are in a bound state caused by capturing processes, the observed higher binary frequency for massive stars compared to solar-mass stars must reflect therefore, at least partly, the presence of these transient capture-formed binaries.

The value of $10-15 \%$ can be regarded as a lower limit, because most, if not all, stars in the cluster are initially surrounded by discs. The large radii of the discs compared to the stellar size results in a much larger cross-section for encounters, an effect that has not been included here. The larger capture cross-section will influence the overall dynamics, leading to more bound systems. Moeckel \& Bally (2007a,b) studied the disc-assisted capture formation of binaries for stars of $M_{1}^{*}=20 M_{\odot}$ and estimated that in the Trapezium region, capture could account for $\sim 50 \%$ of massive stars having a companion after 1 Myr. Since the presence of discs around the stars increases the capture crosssection, it is even possible that the fraction of primordial binaries is the same for solar-mass stars as for massive stars, and that the 
observed higher binarity could be exclusively due to the encounter-induced capture processes. To clarify this, future modelling of the cluster dynamics should include the effect of discs by some means.

However, it is most likely that discs only influence capture processes significantly in the very early cluster development, because observational examples for discs around massive stars are usually only found for young, still embedded, protostars (Cesaroni et al. 1999; Tafoya et al. 2004; Patel et al. 2005; Kraus \& Hillenbrand 2007). Massive stars in a more evolved stage, like in the Trapezium, are usually not surrounded by discs. Processes like photoevaporation (Clarke 2007) and gravitational interaction (Pfalzner et al. 2006) are responsible for the disc destruction. Observational and theoretical evidence point to lifetimes of discs around massive stars of approximately $\sim 10^{5}-10^{6}$ years.

The second question, whether the observed properties of these TBS are in accordance with observations, is difficult to answer. The reason for this is that the ONC contains only 15 OB-stars, and as we have shown, the observed binaries are a combination of primordial binaries and those formed in capture processes. We would expect 2-4 of the massive stars to be part of a TBS - not a sufficient number for statistical purposes. Another problem is that, when observing the Trapezium, the surface density of stars is such that the visual binary population at periastra of a few $100 \mathrm{AU}$ is difficult to distinguish from unassociated stars using current spectroscopic methods. However, future interferometric observations of proper motions - with, for example the VLTI, - should be able to provide the neccessary data.

In this context, it is interessting that recent observations (Kraus et al. 2007) indicate that $\theta^{1} \mathrm{C}$ Ori itself is a binary with a massive companion, possibly with $\theta^{1} \mathrm{C}$ Ori having a mass of $34 M_{\odot}$ and the companion mass being $15.5 M_{\odot}$. Naturally one cannot decide whether a specific binary has formed primordially or as a result of a capture process. In this specific case the high mass ratio would suggest a capture process, but the low periastron of $\sim 1.5$ AU could occur in both cases - capture-formed or primordial binaries.

Although the ONC has the advantage that many observational results are available, it has the disadvantage that it does not contain a high number of massive stars. However, statistical properties in other clusters - especially in very young ones - could provide signatures of capture processes. Properties where one would expect observable differences between primordial and capture-formed binaries are the periastron and the mass ratio distribution. Our simulations show that the periastra of the encounter-induced bound systems are usually in the range of a few 10-2000 AU for the first Myrs of evolution, so they clearly belong to the so-called wide binaries. The observed over-abundance of wide binaries among PMS stars compared to field stars (Patience et al. 2002) could possibly be a tracer for these capture-formed binaries. As we have shown here, the average periastra in TBS with a massive primary becomes smaller $(<100 \mathrm{AU})$ for older clusters, so one should be able to trace this effect by observing the binary frequency of massive stars in clusters of different age but similar structure. The over-abundance of wide binaries should shift to smaller periastron values and become less pronounced with increasing age. The latter is due to the shorter lifetime of the massive stars, so these binaries would naturally disappear if the cluster is beyond a certain age.

Our simulations indicate that capture processes lead to an increase in the mass ratio with cluster age. Investigations of the mass ratio in binaries with massive primaries give a complex picture: while small $q$ (consistent with random pairing) are observed in the ONC (Preibisch et al. 1999), in rich clusters most
O stars are believed to exist as short-period binaries with $q \simeq 1$ (Garcia \& Mermilliod 2001). Mass-ratio distributions not consistent with random pairing were also reported by Kouwenhoven (2005) for A and late-type B binaries in Scorpius OB2.

In future, this kind of investigation should ideally be performed for a cluster containing a high number of $\mathrm{O}$ stars. The question is: what can one expect in systems other than the ONC? In lower density and open clusters the number of bound systems formed by capturing will be too insignificant to contribute to the total binary fraction. However, in high-density clusters like the Arches cluster near the Galactic centre, the central mass density $\left(\sim 3 \times 10^{5} M_{\odot} \mathrm{pc}^{-3}\right.$, Figer et al. 2002) is about ten times higher than in the Trapezium region, so capturing encounters could play an important role. However, this is difficult to predict because a number of other cluster properties influence the capture rate. Apart from the cluster density, the velocity dispersion and the degree of mass segregation will be significant factors. In the Arches cluster the velocity dispersion is not very well known, only an upper limit of $22 \mathrm{~km} \mathrm{~s}^{-1}$ exists. If the real velocity dispersion is close to this upper limit, it might mean a large number of high velocity encounters, which would decrease the capture rate. The relative importance of these different cluster properties for the capture rate need further investigation.

Since the primordial binary fraction seems to depend strongly on the cluster environment, it is important to compare relative values. One way to find indications for the captureformed massive binaries would be to compare the relative binary frequency of solar-type and massive stars in clusters of different stellar density. If capturing is an important process, the higher frequency of capturing encounters in dense systems would lead to a higher proportion of massive binaries to solar-type binaries there. However, one has to be careful with very dense systems containing many OB-stars, since higher velocity dispersion could counteract this process.

The study performed here can only be regarded as a first step towards understanding the significance of capture processes in young dense clusters. In future, some points need further investigation. First, we concentrated here on the closest bound partner, whereas observations, as well as our simulations, indicate that multiple systems are a common feature for massive stars. Second, simulations should start with a finite primordial binary fraction and see how it develops. The massive stars would still capture partners, but it is also known that the dynamical evolution of dense clusters has a destructive effect on primordial binaries (Kroupa 1995; Kroupa et al. 1999; Kroupa 2001). It would be important to see which of these competing effects dominates. Third, as pointed out above, in order to improve the statistics, simulation of systems containing a higher number of OB stars is required. Finally, disc effects have to be included as, despite their short lifetimes around massive stars, they could not only increase the capture-induced binary rate but also influence the periastron and eccentricity of the formed systems. From our results it is apparent that it is not uncommon for the periastron in capture-formed bound systems to be just a few $10 \mathrm{AU}$. This means that the secondary star would pass through the disc of the primary. Repeated disc passages can reduce the periastron and eccentricity through gravitational drag. For our simulations this would mean an even faster development in the initial stages.

Acknowledgements. We are grateful to R. Spurzem for providing the Nbody6++ code for the cluster simulations and want to thank H. Zinnecker for his very helpful comments. Simulations were partly performed at the John von Neumann Institute for Computing, Research Centre Jülich, Project HKU14. 


\section{References}

Aarseth, S. 2003, Gravitational N-Body Simulations (Cambridge: CUP)

Abt, H. A., Gomez, A. E., \& Levy, S. G. 1990, ApJS, 74, 551

Beck, T. L., Simon, M., \& Close, L. M. 2003, ApJ, 583, 358

Boss, A. P. 1992, in The Realm of Interacting Binary Stars, ed. J. Sahade, G. E. McCluskey, \& Yoji Kondo, 355

Bouvier, J., Rigaut, F., \& Nadeau, D. 1997, A\&A, 323, 139

Cesaroni, R., Felli, M., Jenness, T., et al. 1999, A\&A, 345, 949

Clarke, C. J. 2007, MNRAS, 376, 1350

Clarke, C. J., \& Pringle, J. E. 1991, MNRAS, 249, 584

Duchêne, G., Bouvier, J., \& Simon, T. 1999, A\&A, 343, 831

Duchêne, G., Bouvier, J., Bontemps, S., Andre, P., \& Motte, F. 2004, A\&A, 427, 651

Duquennoy, A., \& Mayor, M. 1991, A\&A, 248, 485

Durisen, R. H., \& Sterzik, M. F. 1994, A\&A, 286, 84

Figer D. F., et al. 2002, ApJ, 581, 258

Fischer, D. A., \& Marcy, G. W. 1992, ApJ, 396, 178

Garcia, B., \& Mermilliod, J. C. 2002, A\&A, 386, 122

Goodwin, S. P., Kroupa, P., Goodman, A., \& Burkert, A. 2007, in Protostars and Planets V, ed. D. Reipurth, D. Jewitt, \& K. Keil (Tucson: Univ. Arizona Press), 133

Heggie, D. C. 1975, MNRAS, 173, 729

Heggie, D. C., \& Hut, P. 2003, The Gravitational Million-Body Problem: A Multidisciplinary Approach to Star Cluster Dynamics (Cambridge: CUP)

Hills, J. G. 1975, AJ, 80, 809

Hillenbrand, L. A., \& Hartmann, L. W. 1998, ApJ, 492, 540

Köhler, R., Petr-Gotzens, M. G., McCaughrean, M. J., et al. 2006, A\&A, 458, 461

Kouwenhoven, M. B. N., Brown, A. G. A., Zinnecker, H., Kaper, L., \& Portegies Zwart, S. F. 2005, A\&A, 430, 137

Kraus, A. M., \& Hillenbrand, L. A. 2007, ApJ, 662, 413

Kraus S., Balega, Y. Y., Berger, J.-P., et al. 2007, A\&A, 466, 649

Kroupa, P. 1995, MNRAS, 277, 1491

Kroupa, P. 2001, MNRAS, 322, 231

Kroupa, P., Tout, C. A., \& Gilmore, G. 1993, MNRAS, 262, 545
Kroupa, P., Petr, M., \& McCaughrean, M. 1999, New Astron., 4, 495

Liu, W. M., Meyer, M. R., Cotera, A. S., \& Young, E. T. 2003, AJ, 126, 1665

Luhman, K. L., McLeod, K. K., \& Goldenson, N. 2005, ApJ, 623, 1141

Mason, B. D., Gies, D. R., Hartkopf, W. I., et al. 1998, AJ, 115, 821

Mathieu, R. D. 1994, ARA\&A, 32, 465

Mathieu, R. D., Ghez, A. M., Jensen, E. L. N., \& Simon, M. 2000, in Protostars and Planets IV, ed. V., Mannings, A. P., Boss, \& S. S., Russell (Univ. Arizona Press)

McCaughrean, M. J. 2001, in The Formation of Binary Stars, ed. H. Zinnecker, $\&$ R. D. Mathieu, ASP Conf. Ser., Proc. IAU Symp., 211, 311

McCaughrean, M., Zinnecker, H., Andersen, M., Meeus, G., \& Lodieu, N. 2002, The Messenger, 109, 28

Moeckel, N., \& Bally, J. 2007, ApJ, 656, 275

Moeckel, N., \& Bally, J. 2007, ApJ, 661, L183

Olczak, C., Pfalzner, S., \& Spurzem, R. 2006, ApJ, 642, 1140

Padgett, D. L., Strom, S. E., \& Ghez, A. 1997, ApJ, 477, 705

Patel, N. A., Curiel, S., Sridharan, T. K., et al. 2005, Nature, 437, 109

Patience, J., Ghez, A. M., Reid, I. N., Weinberger, A. J., \& Matthews, K. 1998, AJ, 115, 1972

Patience, J., Ghez, A. M., Reid, I. N., \& Matthews, K. 2002, AJ, 123, 1570

Petr, M. G., Coude de Foresto, V., Beckwith, S. V. W., Richichi, A., \& McCaughren, M. J. 1998, ApJ, 500, 825

Pfalzner, S., Olczak, C., \& Eckart, A. 2006, A\&A, 454, 811

Preibisch, T., Balega, Y., Hofmann, K.-H., Weigelt, G., \& Zinnecker, H. 1999, New Astron., 7, 531

Prosser, C. F., Stauffer, J. R., Hartmann, L., et al. 1994, ApJ, 421, 517

Reid, I. N., \& Gizis, J. E. 1997, AJ, 113, 2246

Scally, A., Clarke, C., \& McCaughren, M. J. 1999, MNRAS, 306, 253

Simon, M., Close, L. M., \& Beck, T. L. 1999, AJ, 117, 1375

Spurzem, R. 1999, Comp. Astroph., 109, 407

Sterzik, M. F., Durisen, R. H., \& Zinnecker, H. 2003, A\&A, 411, 91

Tafoya, D., Gomez, Y., \& Rodriguez, L. F. 2004, ApJ, 610, 827

Tohline, J. E., Cazes, J. E., \& Cohl, H. S. 1999, in Numerical Astrophysics,

Astrophysics and Space Science Library 240, ed. S. M., Miyama, K., Tomisaka, \& T., Hanawa, 155

Yorke, H. W., \& Zinnecker, H. Z., Ann. Rev. Astron. Astrophys., in press 